\title{
Propiedades mecánicas de cermets basados en diboruro de titanio
}

\author{
J.M. SÁNCHEZ ${ }^{(1,2)}$, I. AZCONA ${ }^{(1)}$ Y F. CASTRO ${ }^{(1,2)}$ \\ (1) Centro de Estudios e Investigaciones Técnicas de Guipúzcoa (CEIT) \\ Departamento de Materiales. Paseo Manuel de Lardizábal, 15. 20018. P.O.1555. San Sebastián. \\ (2) Escuela Superior de Ingenieros Industriales. Universidad de Navarra. Apdo. 1674, 20080. San Sebastián.
}

\begin{abstract}
Las propiedades mecánicas de los cermets basados en diboruro de titanio $\left(\mathrm{TiB}_{2}\right)$ dependen críticamente de la composición de la fase ligante. Se ha comprobado que tanto la tenacidad como la dureza aumentan significativamente si se evita la formación de boruros secundarios durante la sinterización en fase líquida. Las observaciones fractográficas realizadas en cermets basados en $\mathrm{TiB}_{2}$ sin boruros secundarios confirman el comportamiento plástico de la fase ligante durante la fractura. La ruta pulvimetalúrgica aplicada a estos materiales permite la modificación intencionada de la estructura de la fase ligante desde ferrita a austenita mediante adiciones de aluminio a las mezclas de polvos. Los valores de tenacidad más elevados se han obtenido para los cermets con matriz austenítica. El análisis mediante difracción de rayos X de la superficie de fractura de estos materiales confirma que la fase ligante experimenta transformación martensítica durante la fractura, mecanismo de aumento de tenacidad ya observado en otros sistemas. Esta nueva familia de materiales duros presenta una excelente combinación de dureza y tenacidad, comparable a la obtenida con grados comerciales de carburos cementados (WC-Co).
\end{abstract}

Palabras clave: Diboruro de titanio, cermets, dureza, tenacidad, transformación martensítica.

\section{Mechanical properties of titanium diboride based cermets}

Mechanical properties of titanium diboride $\left(\mathrm{TiB}_{2}\right)$ cermets critically depend on the composition of the binder phase. Both, fracture toughness and hardness, are substantially increased by avoiding the formation of extremely brittle secondary borides formed during sintering by chemical reactions between $\mathrm{TiB}_{2}$ and the metallic additives. Fractographic observations of $\mathrm{TiB}_{2}$ cermets without secondary borides show the presence of ductile ligaments of the binder phase bridging the advancing crack tip. The powder metallurgy processing route applied to these materials allows modification of the binder phase structure from the ferritic iron-aluminium phase to Fe-Ni-Al austenite by changing the aluminium content of the powder mixtures. The highest toughness values have been obtained for the $\mathrm{TiB}_{2}$ cermets with an austenitic binder phase. X-ray diffraction analyses of the fracture surfaces of such samples show that the binder phase is metastable exhibiting stress induced martensitic transformation during fracture. This new family of materials presents an outstanding combination of hardness and toughness, comparable to those obtained with commercial grades of tungsten carbide (WC) hardmetals.

Keywords: titanium diboride, cermets, hardness, toughness, martensitic transformation

\section{INTRODUCCIÓN}

El refuerzo mediante el uso de matrices metálicas ha sido una idea comúnmente utilizada no sólo para aumentar la tenacidad del diboruro de titanio $\left(\mathrm{TiB}_{2}\right)$ sino también su sinterabilidad (1-5). Metales como el hierro, el cobalto o el níquel fueron los primeros utilizados para la sinterización en fase líquida del $\mathrm{TiB}_{2}$. Sin embargo, estos metales de transición reaccionan con el diboruro de titanio para formar boruros metálicos del tipo $\mathrm{MB}, \mathrm{M}_{2} \mathrm{~B}$ y $\mathrm{M}_{23} \mathrm{~B}_{6}$ (con $\mathrm{M}=\mathrm{Fe}, \mathrm{Ni}, \mathrm{Co}, \mathrm{Ti}$ ), que son incluso más frágiles que el propio $\mathrm{TiB}_{2}(5)$. Trabajos recientes (6-9) han demostrado que ciertas aleaciones de hierro y níquel con adiciones controladas de titanio y aluminio pueden usarse como ligantes para el $\mathrm{TiB}_{2}$, ya que inhiben la formación de estos boruros secundarios. La composición química de estas aleaciones, estables en fase líquida con el diboruro de titanio, han sido determinadas mediante cálculos termodinámicos (9). Siguiendo esta ruta se han producido diversos cermets basados en $\mathrm{TiB}_{2}$ con fases ligantes metálicas e intermetálicas. El objetivo de este trabajo es presentar las propiedades mecánicas más significativas de estos nuevos cermets: dureza, resistencia a la flexión, módulo de elasticidad y, especialmente, tenacidad además de discutir los mecanismos de refuerzo observados para las diversas fases intergranulares.
TABLA I. COMPOSICIONES (\% EN PESO) DE LAS TRES ALEACIONES USADAS COMO LIGANTES DEL TiB 2

\begin{tabular}{|ll|l|l|l|}
\hline Composiciones & $\mathrm{Fe}$ & $\mathrm{Ni}$ & $\mathrm{Ti}$ & $\mathrm{Al}$ \\
\hline FN & 70 & 30 & - & - \\
\hline FNTA (1) & 50.5 & 21.5 & 10.4 & 17.6 \\
\hline FNTA (2) & 58.0 & 25.0 & 6.3 & 10.7 \\
\hline
\end{tabular}

\section{PROCEDIMIENTO EXPERIMENTAL}

\subsection{Materiales}

De acuerdo con los resultados obtenidos en anteriores trabajos $(9,10)$, se han seleccionado tres aleaciones metálicas para estudiar su efecto sobre las propiedades mecánicas de los cermets basados en $\mathrm{TiB}_{2}$ (Tabla I). Los contenidos de fase ligante seleccionados para dichos cermets oscilan entre $8 \%$ y $30 \%$ en volumen. 


\subsection{Propiedades mecánicas.}

Los ensayos de dureza Vickers se realizaron sobre cilindros de $13 \mathrm{~mm}$ de diámetro y $5 \mathrm{~mm}$ de altura. Los resultados presentados corresponden a la media de 5 ensayos realizados con un indentador Tukon a una carga de $10 \mathrm{~kg}$ (HV10). Las grietas generadas en las esquinas de las huellas de indentación se usaron para calcular el factor crítico de intensidad de tensiones superficial, $\mathrm{K}_{\mathrm{IC}}^{\mathrm{S}}$ mediante el modelo semi-empírico propuesto por Shetty et al. (11):

$$
K_{I C}^{S}=\beta\left[\frac{H\left(P-P_{c}\right)}{4 a}\right]^{1 / 2}
$$

donde, $\mathrm{P}$ es la carga aplicada, $\mathrm{P}_{\mathrm{c}}$ es la carga umbral para el inicio del agrietamiento, $\mathrm{H}$ es la dureza Vickers, b es una constante que depende de la geometría del indentador y que en el caso de la pirámide Vickers vale 0.089 y, finalmente, "a" es el tamaño de la grieta considerada como tipo Palmqvist (12). No se observó agrietamiento para contenidos de fase ligante iguales o superiores al $30 \%$ en volumen incluso para cargas de 25 $\mathrm{kg}$. La medida directa de tenacidad de los cermets basados en $\mathrm{TiB}_{2}$ se realizó mediante el ensayo Barker de acuerdo con la norma ASTM E1304-89 (13). Las probetas fueron producidas mediante compresión isostática en caliente (HIP) en forma de cilindros de $15 \mathrm{~mm}$ de altura y $12 \mathrm{~mm}$ de diámetro, y, posteriormente, mecanizadas por electroerosión. Se ensayaron 5 muestras para cada composición usando una máquina convencional de ensayos con una velocidad del actuador de 0.1 $\mathrm{mm} / \mathrm{min}$. La fórmula utilizada para evaluar $\mathrm{K}_{\mathrm{IC}}$ es:

$$
K_{I C S R}=\frac{Y^{*} P_{\max }}{B \sqrt{W}}
$$

donde, $\mathrm{Y}^{*}$ es un factor geométrico cuyo valor es $29.21, \mathrm{P}_{\max }$ es la carga máxima aplicada y $\mathrm{B}$ y $\mathrm{W}$ son el diámetro y la longitud del cilindro respectivamente.

La caracterización microstructural de los materiales sinterizados se realizó mediante microscopía electrónica de barrido sobre muestras pulidas hasta un acabado de pasta de diamante de $1 \mu \mathrm{m}$.

\section{RESULTADOS}

\subsection{Microstructuras}

Las microestructuras típicas de los cermets basados en $\mathrm{TiB}_{2}$ están constituidas mayoritariamente por los granos de $\mathrm{TiB}_{2}$ que aparecen rodeados por la fase intergranular (Fig. 1)(14). Todas las muestras sinterizadas presentan una fina dispersión de partículas de alúmina en la fase ligante (fase oscura en la fig. 1). Estas inclusiones son producto del proceso de desoxidación "in situ" que ocurre durante la sinterización. Desde el punto de vista del comportamiento mecánico de los cermets basados en $\mathrm{TiB}_{2}$, el hecho más importante es que la naturaleza de la fase ligante puede modificarse intencionadamente mediante adiciones de titanio y aluminio a las mezclas de polvos. En este trabajo se demostró que la aleación denominada FN produce al sinterizar con $\mathrm{TiB}_{2}$ una fase intergranular compuesta mayoritariamente por el boruro tipo $\mathrm{M}_{2} \mathrm{~B}$ (con $\mathrm{M}=$ $\mathrm{Fe}, \mathrm{Ni}$ ) con restos de austenita. Las adiciones de aluminio y titanio evitan la formación del boruro $\mathrm{M}_{2} \mathrm{~B}$. La aleación FNTA

\begin{tabular}{|c|c|c|c|c|c|c|c|}
\hline \multirow{2}{*}{$\begin{array}{c}\text { Composición } \\
\text { fase ligante } \\
\text { (Tabla 1) }\end{array}$} & \multirow[t]{2}{*}{$\begin{array}{l}\text { Estructura } \\
\text { cristalina }\end{array}$} & \multirow{2}{*}{$\begin{array}{c}\text { Fracción } \\
\text { vol. } \\
\text { ligante } \\
(\%)\end{array}$} & \multirow[t]{2}{*}{$\begin{array}{c}\text { of } \\
(\mathrm{MPa})\end{array}$} & \multirow[t]{2}{*}{$\underset{(\mathrm{GPa})}{\mathrm{E}}$} & \multirow[t]{2}{*}{$\begin{array}{l}\mathrm{HV} 10 \\
\text { (GPa) }\end{array}$} & \multicolumn{2}{|c|}{$\begin{array}{c}\mathrm{K}_{\mathrm{Ic}} \\
\text { (MPa. } / \mathrm{m})\end{array}$} \\
\hline & & & & & & $\begin{array}{c}\text { Test de } \\
\text { indentación }\end{array}$ & BARKER \\
\hline $\mathrm{FN}$ & $\mathrm{M}_{2} \mathrm{~B}$ & 20 & $435 \pm 78$ & $325 \pm 40$ & $16.9 \pm 0.5$ & $5.5 \pm 0.8$ & - \\
\hline \multirow{5}{*}{ FNTA (1) } & \multirow{5}{*}{$\beta_{2}$ - ferrita } & 8 & & 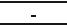 & $20.0 \pm 1.0$ & $8.2 \pm 0.3$ & - \\
\hline & & 14 & & & $18.7 \pm 0.6$ & $11.2 \pm 0.5$ & $9.0 \pm 0.8$ \\
\hline & & 20 & $1096 \pm 70$ & $405 \pm 31$ & $17.8 \pm 0.4$ & $12.3 \pm 0.6$ & $8.0 \pm 1.0$ \\
\hline & & 25 & - & - & $14.6 \pm 1.0$ & $15.2 \pm 0.7$ & $12.3 \pm 1.5$ \\
\hline & & 30 & $1221 \pm 93$ & $396 \pm 27$ & $14.9 \pm 0.3$ & * & $10.0 \pm 1.3$ \\
\hline FNTA (2) & austenita & 30 & $1014 \pm 90$ & $343 \pm 30$ & $14.5 \pm 0.2$ & * & $14.4 \pm 0.8$ \\
\hline
\end{tabular}

Tabla II. Propiedades mecánicas de los Cermets basados en TiB 2

* No se observan grietas producidas por indentación.

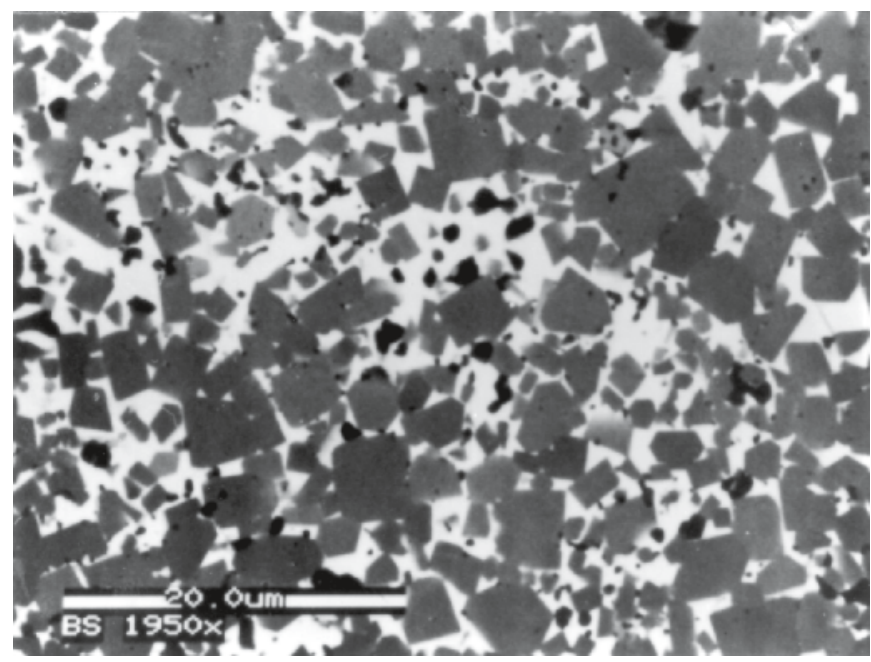

Fig. 1 Micrografía SEM obtenida con electrones retrodispersados. $\mathrm{TiB}_{2}+20 \mathrm{vol} \%$ FNTA(1). Las partículas de alúmina aparecen en contraste oscuro, los granos de $\mathrm{TiB}_{2}$ en gris y la fase ligante en contraste claro.

(1), con alto contenido en $\mathrm{Ti}$ y $\mathrm{Al}$, forma una fase tipo $\mathrm{b}_{2}$ de estructura ferrítica y la aleación FNTA (2) forma austenita (6).

\subsection{Dureza}

Los resultados experimentales confirman que la dureza de los cermets basados en $\mathrm{TiB}_{2}$ puede ser variada intencionadamente si se evitan las reacciones químicas (Tabla II). Dichas reacciones consumen una parte del $\mathrm{TiB}_{2}$ original y la mayoría de las adiciones metálicas para formar los boruros secundarios. La extensión de la reacción depende del contenido de impurezas y es muy difícil de predecir. En la actualidad, se han conseguido durezas de 20 GPa (HV10) para adiciones del 8 vol $\%$ de la aleación FNTA (1). Estos valores son comparables a los de los carburos cementados (WC-Co) producidos a partir de polvos nanométricos (Fig. 2), lo que permite pensar en conseguir durezas aun mayores ya que los polvos de $\mathrm{TiB}_{2}$ producidos hasta la fecha tienen tamaños mucho mayores que los de WC (5 mm frente a $0.3 \mathrm{~mm}$ del WC) $(15,16)$.

\subsection{Tenacidad}

En la Fig. 3, se presentan los datos de dureza y tenacidad correspondientes a los cermets obtenidos con la aleación FNTA(1) (entre $8 \%$ y $30 \%$ en volumen de fase ligante) y la aleación FN (20\% en volumen de fase ligante). Estos datos confirman que la presencia del boruro $\mathrm{M}_{2} \mathrm{~B}$ (aleación $\mathrm{FN}$ ) supone una disminución drástica de la tenacidad acompañada incluso de una disminución ligera de dureza respecto al caso en el que la fase ligante es de 


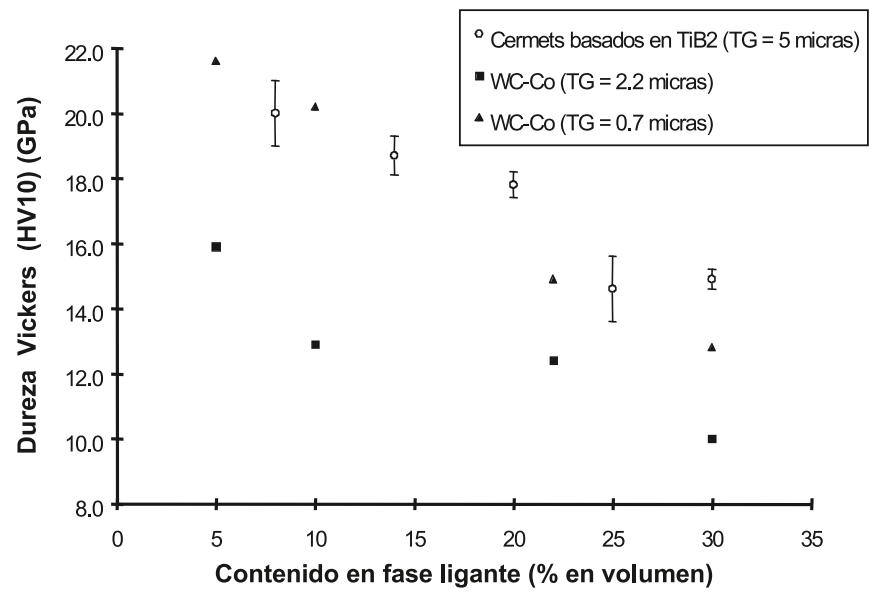

Fig. $2 \mathrm{TiB}_{2}$ + aleación FNTA (1). Dureza Vickers (HV10) vs. fracción en volumen de fase ligante. Se incluyen datos de carburos cementados basados en WC-Co $(15,16)$.

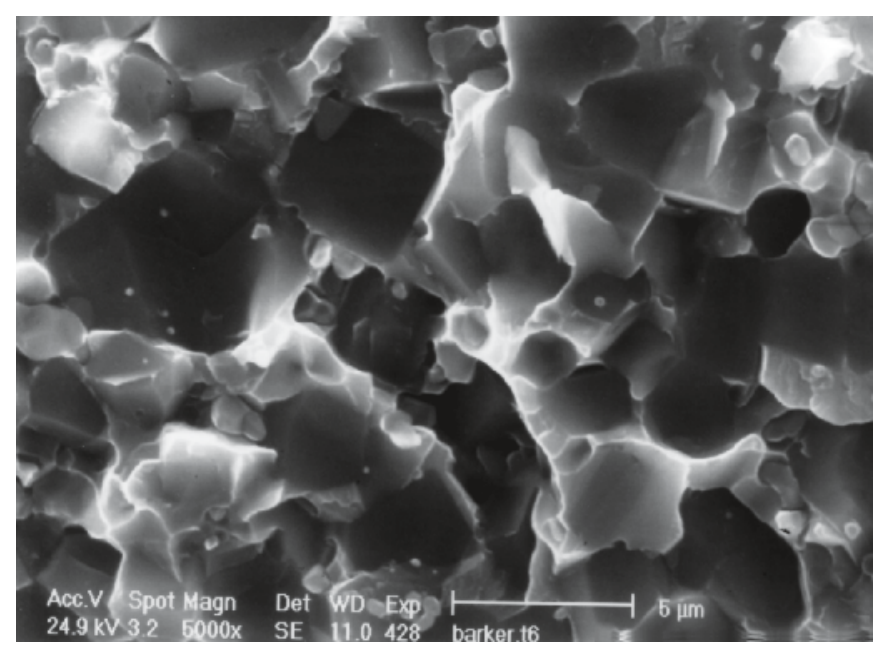

Fig. $4 \mathrm{TiB}_{2}+20 \mathrm{vol} \%$ FNTA(1). Micrografía SEM obtenida mediante electrones secundarios correspondiente a la superficie de fractura de una probeta Barker. En la fase ligante se observan micro-cavidades con partículas de alúmina en su interior y restos de ligamentos plásticos con una significativa reducción en área.

estructura ferrítica tipo $\beta_{2}$ (aleación FNTA(1)). Los análisis fractográficos realizados demuestran que los cermets en los que no aparece el boruro $\mathrm{M}_{2} \mathrm{~B}$ presentan claras similitudes con los carburos cementados (WC-Co) (17). Se pueden distinguir cuatro modos de rotura (Fig. 4): exfoliación de los granos de $\mathrm{TiB}_{2}$, rotura de la frontera de grano $\mathrm{TiB}_{2} / \mathrm{TiB}_{2}$, rotura de la intercara $\mathrm{TiB}_{2} /$ metal y rotura dúctil de la fase ligante. El inicio de la fractura de la fase ligante parece estar relacionado con la formación de microcavidades por descohesión de la intercara alumina-fase ligante, como lo demuestra el hecho de que estas cavidades siempre presentan en su interior partículas de alúmina. Estas observaciones sugieren que la reducción del contenido de oxígeno de los polvos de partida (origen de la formación de las partículas de alúmina) permitiría aumentar la resistencia a la fractura de los cermets basados en $\mathrm{TiB}_{2}$. Esta descripción es compatible con el análisis de las grietas generadas mediante indentación (Fig. 5). La punta de la grieta se propaga a través de los granos de $\mathrm{TiB}_{2} \mathrm{o}$ a lo largo de la intercara $\mathrm{TiB}_{2}$-fase ligante mediante los mecanismos antes descritos. Sin embargo, la fase ligante frena la propagación al defor-

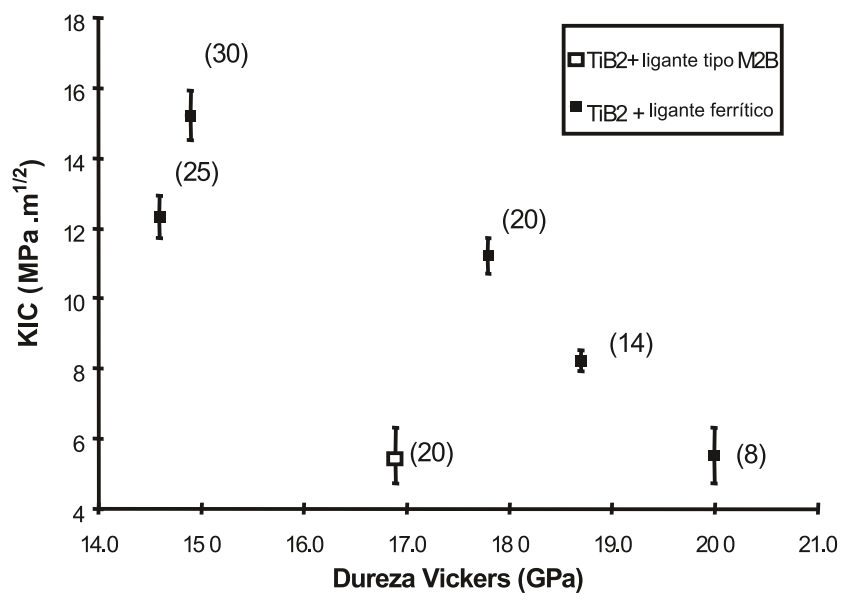

Fig. 3 Cermets basados enTiB 2 . Dureza Vickers frente a tenacidad (KIC). Contenido en fase ligante para cada material entre paréntesis (\% en volumen)

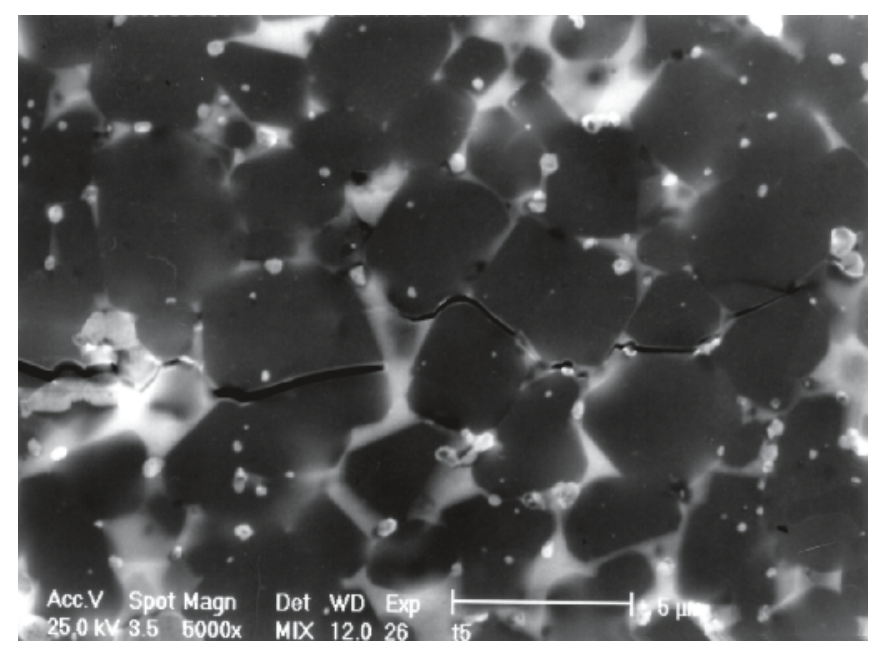

Fig. 5 Composición $\mathrm{TiB}_{2}+20$ vol\% FNTA (1). Grieta producida por indentación. Se observa que la grieta progresa dejando ligamentos de fase matriz sin fracturar.

marse plásticamente formando un conjunto de ligamentos que unen los bordes de la grieta.

Finalmente, los resultados obtenidos mediante el uso de la aleación FNTA(2) abren una ruta alternativa para una mejora adicional de la tenacidad de los cermets basados en $\mathrm{TiB}_{2}$ mediante la aplicación del concepto de transformación martensítica inducida por deformación (18). Los patrones de difracción de rayos $\mathrm{X}(\mathrm{XRD})$ obtenidos de la superficie de fractura de las probetas Barker confirman que el ligante austenítico que se obtiene con la aleación FNTA (2) es metastable y se transforma casi completamente en martensita durante el proceso de fractura. La correlación entre el grado de transformación y el incremento de $\mathrm{K}_{\mathrm{IC}}$ es objeto de investigación en la actualidad. No obstante, resultados preliminares muestran que la tenacidad de estos nuevos cermets es superior a la de los materiales con matriz no transformable. La energía requerida para la transformación se obtiene del campo de tensiones en el entorno de la punta de la grieta, lo que reduce el factor de concentración de tensiones. 


\section{CONCLUSIONES}

Los cermets basados en $\mathrm{TiB}_{2}$ son materiales ultra duros con propiedades comparables a las de los grados comerciales de metal duro $\left(\mathrm{HV} 10 \approx 19 \mathrm{GPa}\right.$ y $\mathrm{K}_{\mathrm{ICSR}} \approx 9 \mathrm{MPa} \cdot \sqrt{\mathrm{m}}$ ). Estos resultados constituyen una mejora significativa respecto a los materiales compuestos basados en $\mathrm{TiB}_{2}$ producidos hasta la fecha. Como se describe en el presente trabajo, el comportamiento plástico de la fase ligante durante la fractura es responsable del incremento de tenacidad observado en estos materiales. Se ha mostrado que las fases ligantes de naturaleza austenítica proporcionan un incremento adicional de tenacidad asociado a mecanismos de transformación martensítica por deformación. La producción de estos materiales exige un estricto control de la composición química de la mezcla de polvos y las condiciones de sinterización para evitar la formación de fases intergranulares frágiles.

\section{AGRADECIMIENTOS}

Los autores agradecen el apoyo económico de la UE a través del proyecto BRITE-EURAM BE96-3356, “ULTRA HARD MATERIALS FOR TRIBOLOGICAL APPLICATION (ULTRATRIBO)"', (Contract No BRPR-CT96-0304).

\section{BIBLIOGRAFÍA}

1. P.A. Dearnley and T. Bell, Surface Engineering, "Engineering the surface with boron based materials", vol.1, No.3, 203-217 (1985).

2. S. Baik and P.F. Becher, "Effect of oxygen contamination on densification of TiB2" J. Amer. Cer. Soc., 70, 8, 527-530, (1987)

3. J.D. Katz, R.D. Blake and C.P. Scherer,"Microwave sintering of titanium dibo- ride", Ceram. Eng. Sci. Proc, 10, 7-8, 857-867, (1989).

4. G.V. Samsonov, "Nature of interaction between titanium diboride and the iron-group materials", Met. Obra. Metall., 1, 35-38, (1958).

5. H. Pastor, "Boron and refractory borides", V.I. Matkovich, Springer-Verlag, Berlin, 457-493, (1955).

6. J.M. Sánchez, M.G. Barandika, J.Gil Sevillano and F.Castro, “Consolidation, microstructure and mechanical properties of newly developed TiB2 based materials", Scr. Metall. Mater., 26, 957-962, (1992).

7. H. Pastor, C. Allibert, M.G. Barandika, J.M. Sánchez, J.Gil, M.Fuentes and F. Castro,"Development of new boride-based cermets and ceramics", Int. Conf. on Advances in Hard Materials Production, Bonn, 135-146, (1992).

8. H. Pastor, C.H. Allibert, M.L. Ottavi, M. Albajar and F. Castro, "Cermets à base de borures des metaux de transition, leur fabrication et leur applications", Patent 91 08030, Paris, (1991).

9. M.G. Barandika, J.M. Sánchez and F. Castro, “New Developments of TiB2based hardmetals", Metal Powder Report, 2, 10-12, (1994).

10. F. Castro and I.Iturriza, "HIP of Si3N4 and Si3N4 + 1w/o Y2O3 to full density", J. Mater.Sci. Lett., 9, 600-602, (1990).

11. K. Shetty, I.G. Wright, P.N. Mincer and A.H.Clauer. "Indentation fracture of WC-Co cermets",J. Mater. Sci., 20, p.1873-1882, (1985).

12. J.M. Sánchez, "Sinterización y propiedades mecánicas de cermets basados en diboruro de titanio." Tesis doctoral. Universidad de Navarra, 91., (1993).

13. "Standard Test Method for Plane-Strain (Chevron-Notch) Fracture Toughness of Metallic Materials", ASTM Designation E 1304-89, 962 (1990).

14. M.G. Barandika, J.M. Sánchez, T. Rojo, R. Cortés and F. Castro."Fe-Ni-Ti binder phases for TiB2-based cermets: a thermodynamic approach", Scripta. Materialia. Vol. 39, No. 10, 1395-1400, (1998).

15. M.T. Laugier, "A microstructural model for hardness in WC-Co composites", Acta metall. Vol. 33, No. 11, 2093-2099, (1985).

16. L.J. Chermant and F. Osterstock, "Fracture toughness and fracture of WC-Co composites", J. Mater. Sci. 11, p.1939-1951, (1976).

17. J. Hong and J. Gurland, "A study of the fracture process of WC-Co alloys", Science of Hard Materials, R.K. Viswanadham, D.J. Rowcliffe and J. Gurland, eds. Plenum Press, New York, 649-669, (1983).

18. R. Telle and G. Petzow,"Strengthening and Toughening of Boride and Carbide Hard Material Composites", Mater. Sci. and Eng., A105/106, 125129, (1988). 